FERMILAB-TM-2756-TD

January 28, 2021

\title{
A WHITE PAPER ON DESIGN AND FABRICATION OF SRF DEFLECTING CAVITIES FOR ELLETRA-2
}

\author{
A. Lunin, T. Khabiboulline, V. Yakovlev \\ FNAL, Batavia, IL, 60510, USA
}

\section{TABLE OF CONTENTS}

\section{Section I}

1.0 Introduction

2.0 Design Concept of SRF Deflecting Cavity for the Trieste SPX Project

\section{Section II}

3.0 Deflecting Cavities Longitudinal and Transverse Impedances

4.0 Analysis of Short Range Wakefields Excitation

5.0 Parameters of the Operating Mode and Cavity Cryogenic Losses

\section{Section III}

6.0 Estimations of the Cost and Duration of the Cavity EM and Mechanical Design Work

7.0 Anticipated Cost of Operation and Maintenance of the Deflecting Cavity System

8.0 Final Remarks and Conclusion

\subsection{Introduction}

Synchrotron Trieste (ST) operates an X-ray light source Elettra and plans to upgrade it to a lowemittance high-brightness X-ray source Elettra-2.0 based on a multi-bend achromat lattice. There is also a significant interest among Elettra users to pursue time-resolved studies of matter choosing the appropriate $\mathrm{X}$-ray pulse duration in the range from 20 picoseconds to a few picoseconds FWHM. Recently, X. Huang (SLAC) and A. Zholents (ANL) performed a study sponsored by ST and proposed modifications to the Elettra-2.0 design that will allow production of picosecond x-ray pulses on many beamlines. They showed that two radio frequency deflecting cavities with slightly different frequencies can be installed in the ring's sector 8 and produce time-dependent orbit deflection to a few dedicated electron bunches without affecting other regular bunches. These special bunches produce X-ray pulses in which transverse position or angle, or both, are correlated with time. The short X-ray pulse is then obtained by using a narrow slit positioned downstream in the beamline. Many technical details of the proposed modification to Elettra-2.0 are discussed in the supplemental report. We present here the design of a multicell superconducting deflecting cavity for the purpose of the ST Short Pulse X-ray (SPX) project. 


\subsection{Design Concept of SRF Deflecting Cavity for the Trieste SPX Project}

Multiple electrodes immersed in a hollow waveguide form a trapped-mode resonator. The transverse components of the electromagnetic field of the trapped dipole mode induce a transverse kick and efficiently deflect charged particles passing through the cavity. We propose a scalable design of a superconducting Quasi-waveguide Multicell Resonator (QMiR) seamlessly connected to a beam vacuum chamber. The cavity is completely open at both ends, which significantly reduces the maximum loaded quality factor of the higher-order modes (HOMs), avoids complex HOM couplers, and thus simplifies the mechanical design of the cavity. The same port is used to feed RF power to the operating mode and to extract the same-order modes (SOMs). The proposed design of the deflecting cavity has low parasitic HOM losses and a higher threshold of beam instability due to HOM excitation, which is critical for operation with a high-intensity beam current.

Initially the QMiR cavity was designed, fabricated, and tested for SPX operation of the ANL APS Upgrade project [1-3]. The original three-cell deflecting cavity operates at 2.815-GHz frequency, providing a 2-MV transverse kick. The bared QMiR cavity was successfully tested at $2 \mathrm{~K}$ in a vertical cryostat and demonstrated a record transverse kick of 2.6 MV [4]. The cavity geometry with overall dimensions are illustrated in Figure 1.
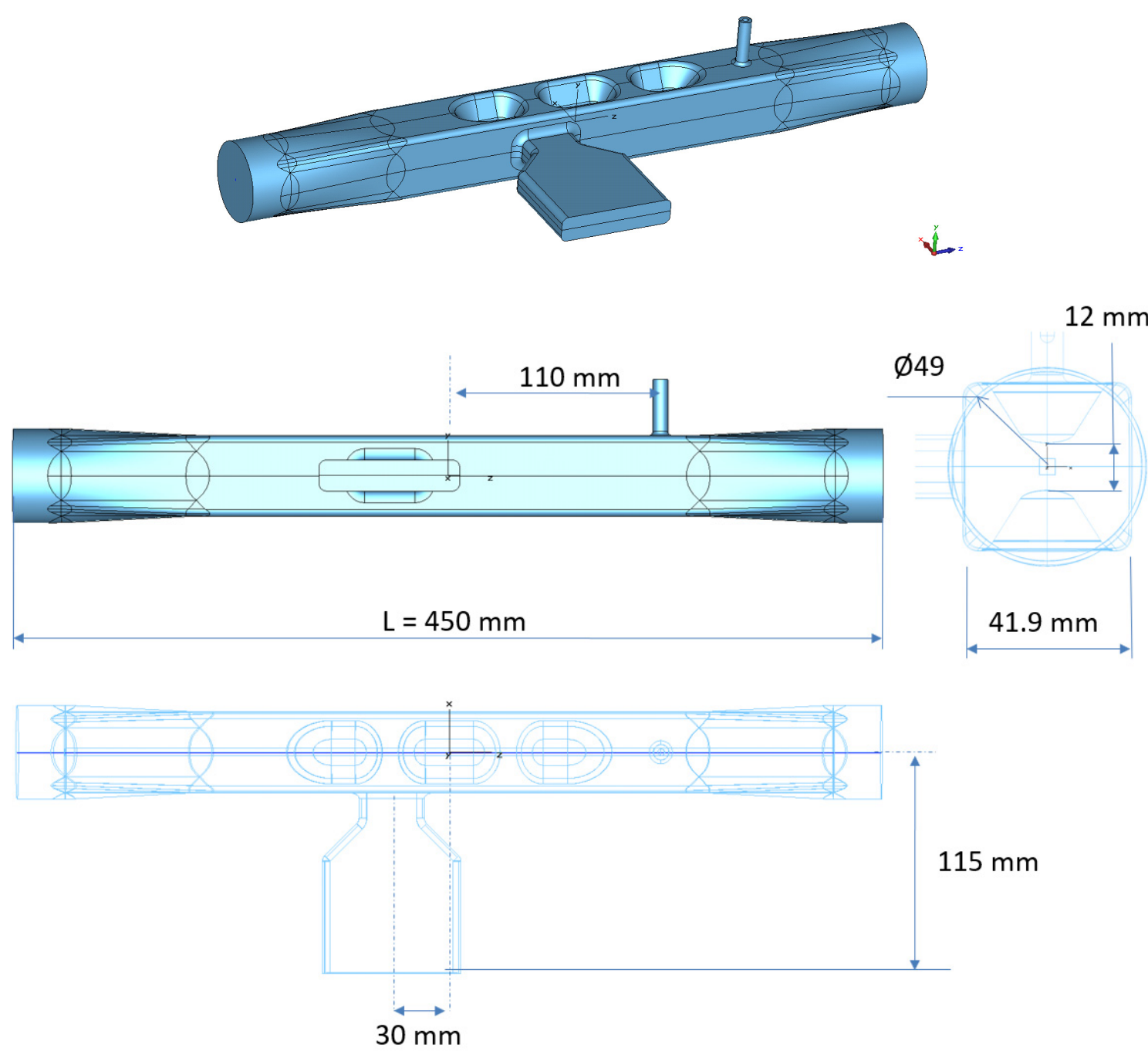

Figure 1. The $2.815 \mathrm{GHz}$ three-cell deflecting cavity for the ANL SPX project. 
Deflecting cavities for the Trieste SPX project are required to operate at $3 \mathrm{GHz}$ and $3.25 \mathrm{GHz}$ frequencies and to provide $0.8 \mathrm{MV}$ and $0.74 \mathrm{MV}$ of total vertical kick, respectively. The easiest and most straightforward way (variant A) to adapt the QMiR design to a new frequency is a uniform scaling of the cavity 3D model. In this case the optimal distribution of operating mode surface fields and the shunt impedance are not changed, preserving the efficiency of a beam deflection. The drawback of this approach is a smaller aperture between electrodes and thus larger cavity loss and kick factors. An alternative option (variant B) is a full redesign of the cavity geometry to a larger beam aperture, aiming to reduce generation of short-range wakefields. We give here estimations for both options of $3-\mathrm{GHz}$ and $3.25-\mathrm{GHz}$ cavities. Geometrical parameters for these two options are listed in Table 1. A comparison of variants A and B of the smallest cavity cross section is shown in Figure 2. Note that in variant B, the operating frequency is adjusted by changing the aperture, while the cavity length remains unchanged.

Table 1. Possible geometrical parameters of Trieste deflecting cavities. Variant A is a scaled version of the $\mathrm{QMiR}$, and variant $\mathrm{B}$ is the QMiR with increased vertical beam aperture.

\begin{tabular}{|l|c|c|c|c|}
\hline \multirow{2}{*}{ Cavity Parameter } & \multicolumn{2}{|c|}{ Variant A } & \multicolumn{2}{c|}{ Variant B } \\
\cline { 2 - 5 } & $3 \mathrm{GHz}$ & $3.25 \mathrm{GHz}$ & $3 \mathrm{GHz}$ & $3.25 \mathrm{GHz}$ \\
\hline Vertical beam aperture, $\mathrm{mm}$ & 11.3 & 10.4 & 13.9 & 16.7 \\
\hline Horizontal beam aperture, $\mathrm{mm}$ & 39.5 & 36.4 & 41.9 & 41.9 \\
\hline Bare cavity length, $\mathrm{mm}$ & 422 & 390 & 450 & 450 \\
\hline
\end{tabular}

Variant $A(3.25 \mathrm{GHz})$

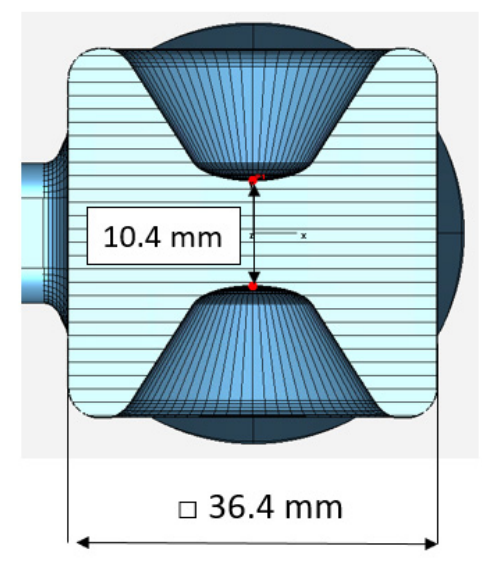

Variant $B(3 \mathrm{GHz})$

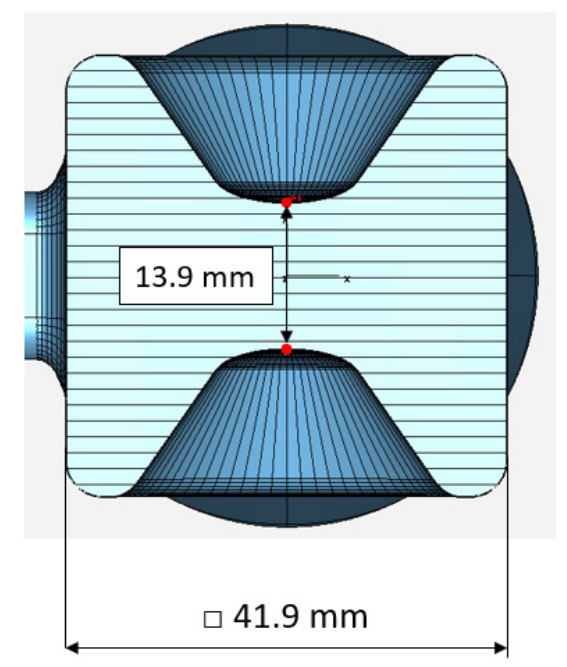

Figure 2. Minimal cavity cross sections of the variant A (left) and variant B (right).

In addition, the original proposal for the Trieste deflecting cavity system is based on a scheme of three cavities where the middle cavity is operating at about double the voltage than the end cavities. Such a scheme is not optimal from the cavity cryogenic loss point of view. It is beneficial to divide the middle cavity into two separate ones, then there will be four cavities in the system, each of which operates at approximately the same voltage. Both schemes are illustrated in Figure 3. A possible coupling between two central cavities operating at the same frequency must be analyzed, and the proper distance between them should be selected to get rid of collective modes. 

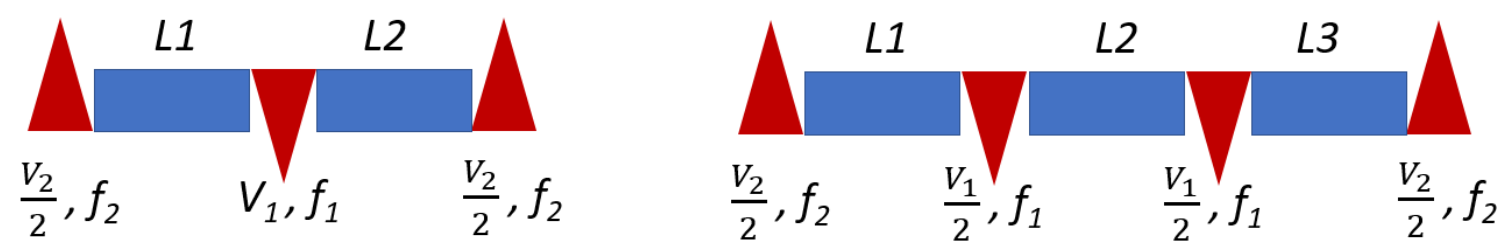

Figure 3. Possible schemes of beam deflecting system for Trieste SPX, using a minimal number of three cavities (left) and four cavities operating at similar voltages (right).

There is a trade-off between minimizing cryogenic losses in the deflecting system, introducing additional parasitic impedances, and creating broadband wakefields. The final selection of the optimal number of deflecting cavities can be made later after a detailed analysis. Cavity spacings are also a subject of optimization for HOM damping, where the minimum spacing can be close to zero, thus making it possible to manufacture all cavities together as one piece from a raw niobium bar. The minimum total length of the deflecting system can be as little as 1 meter and 1.25 meters for three and four cavities, respectively. The proposed complete SRF deflecting systems for ST SPX are shown in Figure 4. The big advantage of making all four cavities as one piece is simplification of the alignment system where alignment of the cavity interconnections is guaranteed with typical machining tolerances of less than 10 microns. Then the coarse alignment can be done by adjusting the cryomodule, and fine tuning and centering of the electron beam is achieved using an intelligent feedback system to maintain the golden orbit.
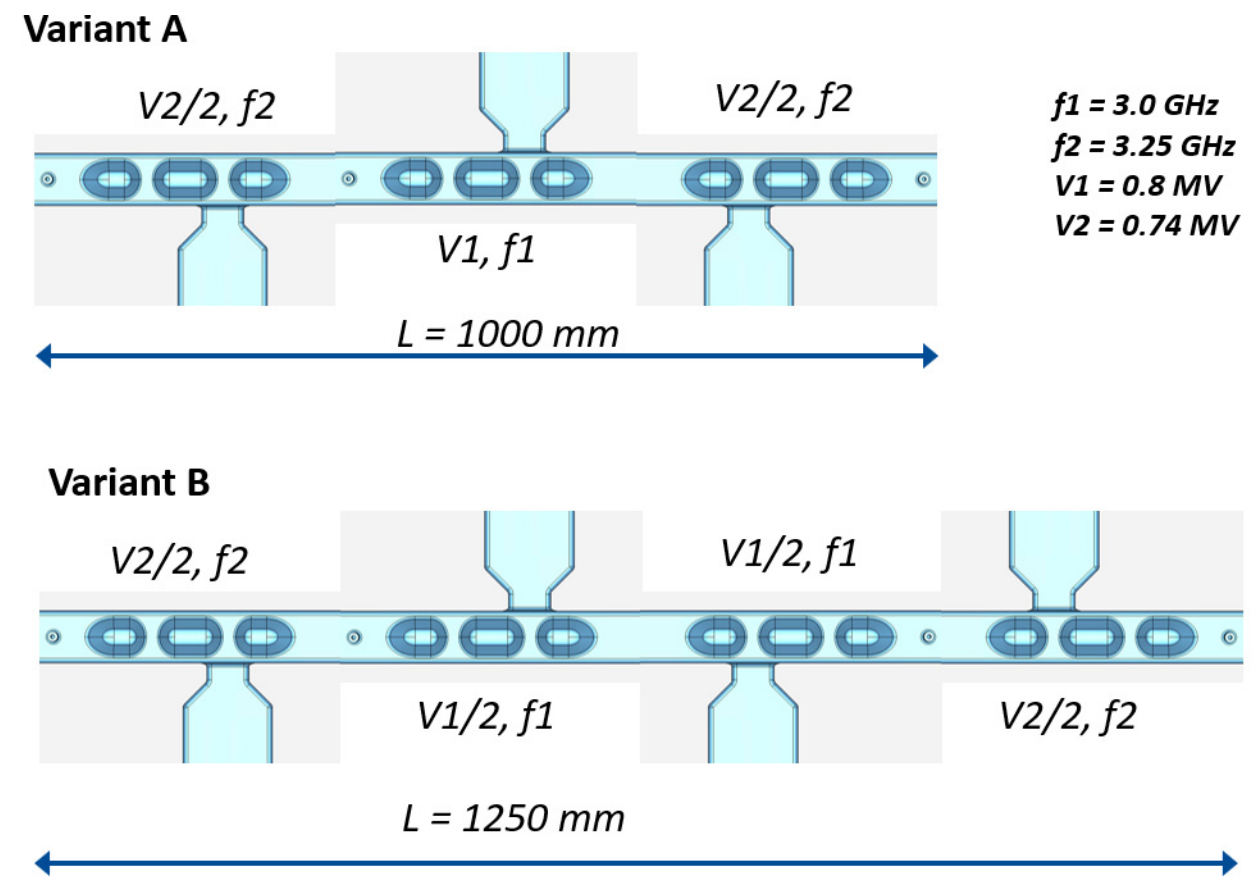

Figure 4. Proposed minimal configurations of SRF deflecting system for ST SPX.

\subsection{Deflecting Cavities Longitudinal and Transverse Impedances}

To analyze the impedance spectrum of the deflecting cavity, we used an ANSYS HFSS eigenmode solver with subsequent post-processing of the field components [5]. The cavity ports are terminated by the broadband Perfect Matched Layer (PML) boundaries, and the external coupling is determined by integrating the Poynting vector flux through the waveguide port. The cavity HFSS model and the electric field map of the operating dipole mode are illustrated in Figure 5. 


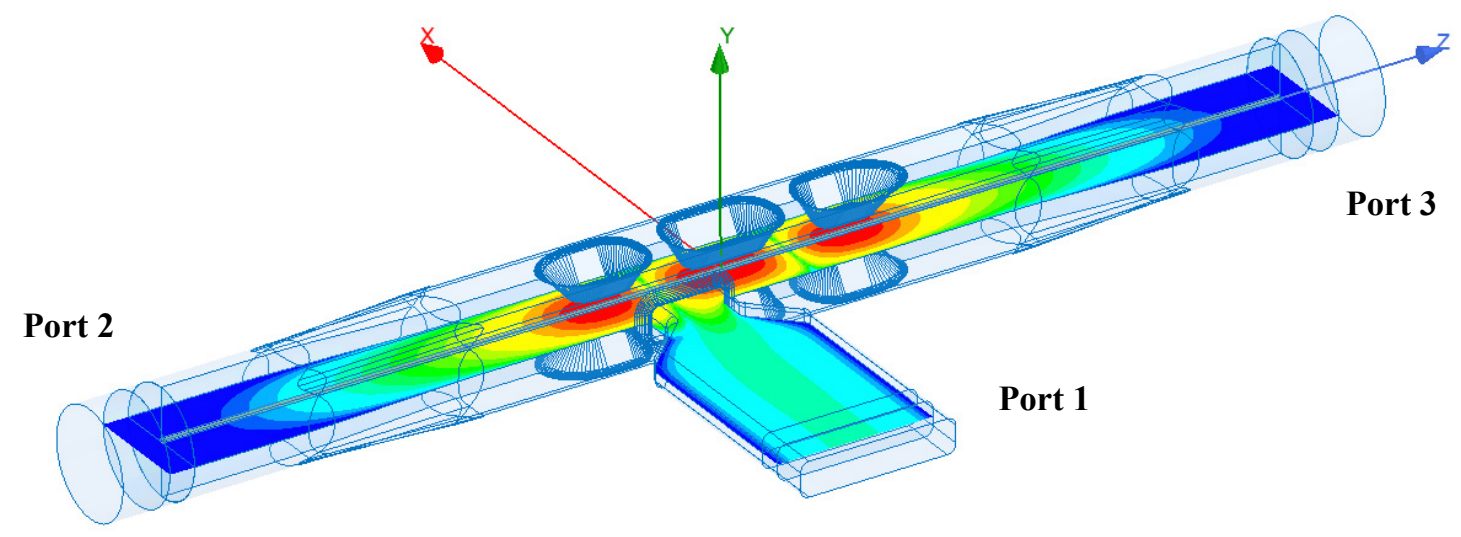

Figure 5. Operating dipole mode in the $3.25 \mathrm{GHz}$ scaled version of the QMiR cavity (logarithmic map of the complex magnitude of the electric field, where a blue color corresponds to zero amplitude).

The lowest frequency eigenmodes of the cavity are the dipole modes. Besides the operational deflecting mode, there are two other SOM deflecting modes whose frequencies are slightly lower. The cut-off frequencies of the lowest dipole $\mathrm{TE}_{11}$ and monopole $\mathrm{TM}_{01}$ modes of the beam pipe are $4.4 \mathrm{GHz}$ and $5.7 \mathrm{GHz}$, respectively for the scaled 3.25-GHz version of the QMiR cavity. Therefore, we limited the eigenmode analysis by an upper frequency of $7 \mathrm{GHz}$. Here we use the circuit definition of a normalized longitudinal shunt impedance for the relativistic particle moving along the cavity axis:

$$
\left(\frac{R_{\|}}{Q}\right)=\frac{\left|\int_{-\infty}^{\infty}\left(E_{z}(r, z)\right)_{r=0} e^{i k z} d z\right|^{2}}{2 \omega_{0} W}
$$

where $\boldsymbol{E}_{\boldsymbol{z}}$ is the longitudinal component of the electric field along the beam trajectory, $r$ is the trajectory radial offset, $W$ is the electromagnetic stored energy, and $\omega_{0}$ is the resonant angular frequency. Similarly, the normalized transverse shunt impedance is defined as:

$$
\left(\frac{R_{\perp}}{Q}\right)=k \frac{\left|\int_{-\infty}^{\infty}\left(\vec{E}_{t}(r, z)+c \vec{z} \times \vec{B}_{t}(r, z)\right)_{r=0} e^{i k z} d z\right|^{2}}{2 \omega_{0} W}
$$

where $\overrightarrow{\boldsymbol{E}}_{\boldsymbol{t}}$ and $\overrightarrow{\boldsymbol{B}}_{\boldsymbol{t}}$ are the corresponding transverse electric and magnetic field components, respectively, and $k$ is the wave number. Computed SOM and HOM shunt impedances and external quality factors for variants $\mathrm{A}$ and B of the ST deflecting cavity are presented in Figures 6 and 7 on the left and the right plots, accordingly. We excluded spurious modes with $\mathrm{Q}_{\mathrm{ext}}<50$, which appeared in simulations due to a slight mismatch of PML boundary conditions. We also slightly tweaked the position of the input waveguide in variant B of the cavity to minimize the resonant excitation of the SOMs. The proposed cavities have sparse spectrums of HOMs due to the nature of the quasi-waveguide loaded to the beam pipe. 

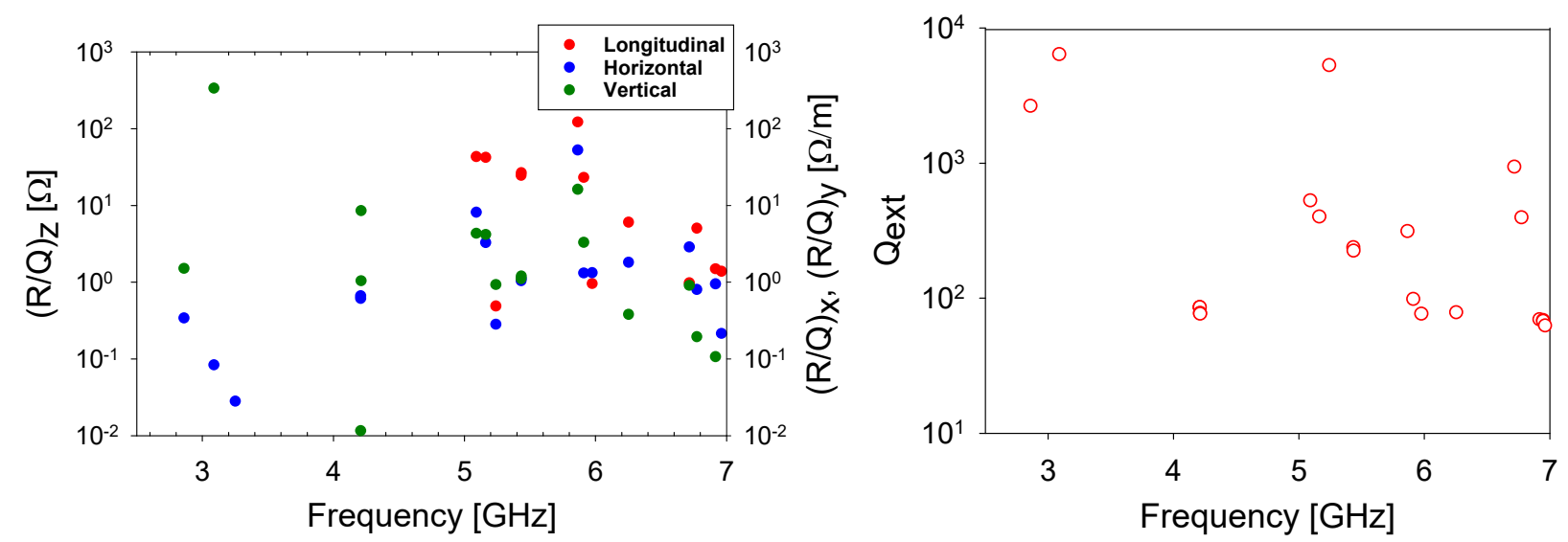

Figure 6. SOM and HOM normalized shunt impedances (left) and external quality factors (right) in the variant A version of the $3.25-\mathrm{GHz}$ QMiR cavity.
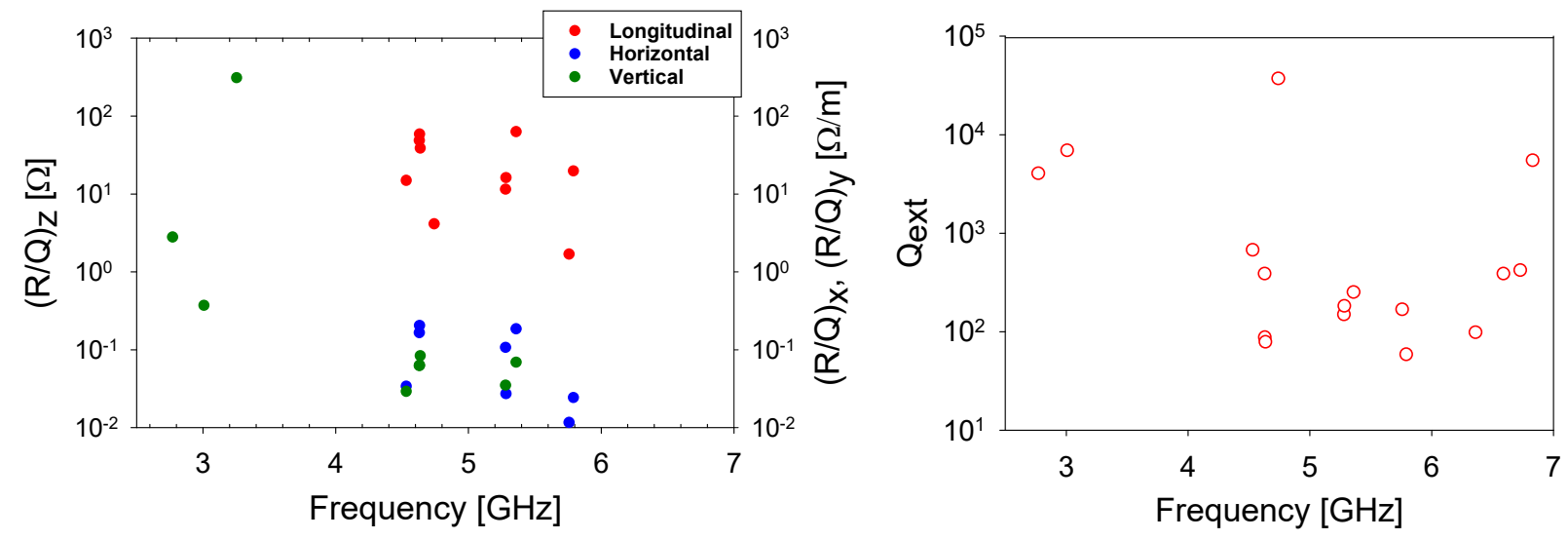

Figure 7. SOM and HOM normalized shunt impedances (left) and external quality factors (right) in the variant B version of the 3.25-GHz QMiR cavity.

Finally, we can estimate the longitudinal and transverse shunt impedances of the most dangerous SOMs and HOMs, considering the exponential form factor $\exp \left(-\omega_{0}{ }^{2} \sigma_{t}^{2}\right)$ of the spectral damping coefficient of a Gaussian bunch with a time length $\sigma_{t}$ as follows:

$$
\begin{aligned}
& R_{\|}=\left(\frac{R_{\|}}{Q}\right) Q e^{-\left(\omega_{0}^{2} \sigma_{t}^{2}\right)}, \\
& R_{\perp}=\left(\frac{R_{\perp}}{Q}\right) Q e^{-\left(\omega_{0}^{2} \sigma_{t}^{2}\right)},
\end{aligned}
$$

Figure 8 shows the shunt impedances of both variants A and $\mathrm{B}$ of the $3.25-\mathrm{GHz}$ deflecting cavity calculated for a $10-\mathrm{mm}$ rms bunch length, where the dashed lines represent the design requirements for the maximum shunt impedances. 

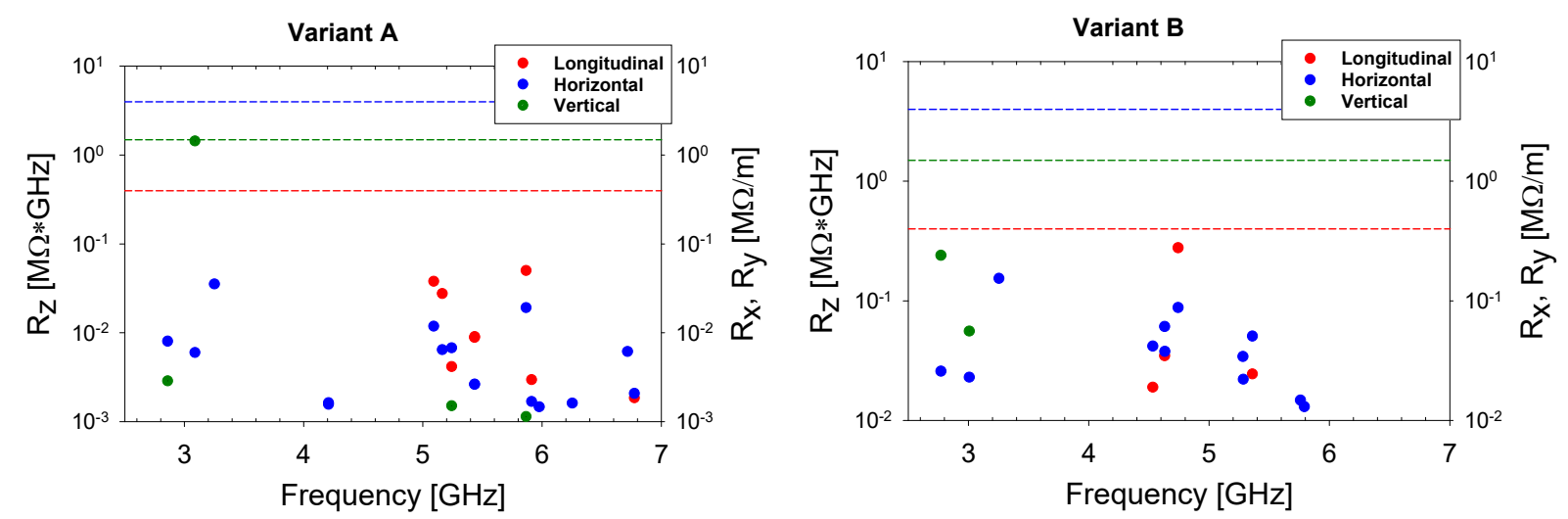

Figure 8. Shunt impedances of variants A (left) and B (right) of the 3.25-GHz deflecting cavity calculated for a 10-mm rms bunch length, where dashed lines are the design requirements for maximum longitudinal (red), vertical (green), and horizontal (blue) shunt impedances.

The final results are summarized in Table 2 for both 3.0-GHz and 3.25-GHz deflecting cavities. One can see that for both variants $A$ and $B$ the maximal cavity shunt impedances are below the limits. The only issue is with the vertical impedance of the second SOM, which is close to the limit for variant A of the cavity. Both variants have room to improve the HOMs damping, specifically by adjusting the cell period and the external coupling with the input rectangular waveguide. We conclude that there is no doubt that both variants of deflecting cavities will meet the impedance requirements set by the ST SPX project.

Table 2. Maximal SOM/HOM shunt impedances of variants A and B of the deflecting cavities.

\begin{tabular}{|l|c|c|c|c|c|}
\hline \multirow{2}{*}{$\begin{array}{l}\text { Maximal SOM/HOM } \\
\text { Shunt Impedance }\end{array}$} & \multicolumn{2}{|c|}{ Variant A } & \multicolumn{2}{c|}{ Variant B } & \multirow{2}{*}{$\begin{array}{c}\text { Project } \\
\text { Limit }\end{array}$} \\
\cline { 2 - 5 } & $3 \mathrm{GHz}$ & $3.25 \mathrm{GHz}$ & $3 \mathrm{GHz}$ & $3.25 \mathrm{GHz}$ & 0.4 \\
\hline Longitudinal, $\mathrm{M} \Omega * \mathrm{GHz}$ & 0.08 & 0.05 & 0.04 & 0.27 & 1.5 \\
\hline Vertical, $\mathrm{M} \Omega / \mathrm{m}$ & 1.3 & 1.4 & 0.32 & 0.24 & 4 \\
\hline Horizontal, $\mathrm{M} \Omega / \mathrm{m}$ & 0.04 & 0.01 & 0.005 & 0.015 & 4 \\
\hline
\end{tabular}

\subsection{Analysis of Short Range Wakefields Excitation}

Relativistic charged particles passing through the cavity excite broadband wakefields that cannot be effectively extracted with conventional HOM couplers; therefore, it is assumed that most of the associated electromagnetic power is absorbed in the upstream and downstream beamline components. We used CST Particle Studio to numerically analyze the wakefield generated by a relativistic Gaussian bunch passing through the cavity (see Figure 9) at a given offset. The result is the longitudinal and transverse wake potentials computed along the axis of the particle beam. Figure 10 shows the wake potentials exited by a bunch with an rms length of $10 \mathrm{~mm}$ and a vertical offset of $1 \mathrm{~mm}$ in variant A of the deflecting cavity with a frequency of $3.25 \mathrm{GHz}$. 


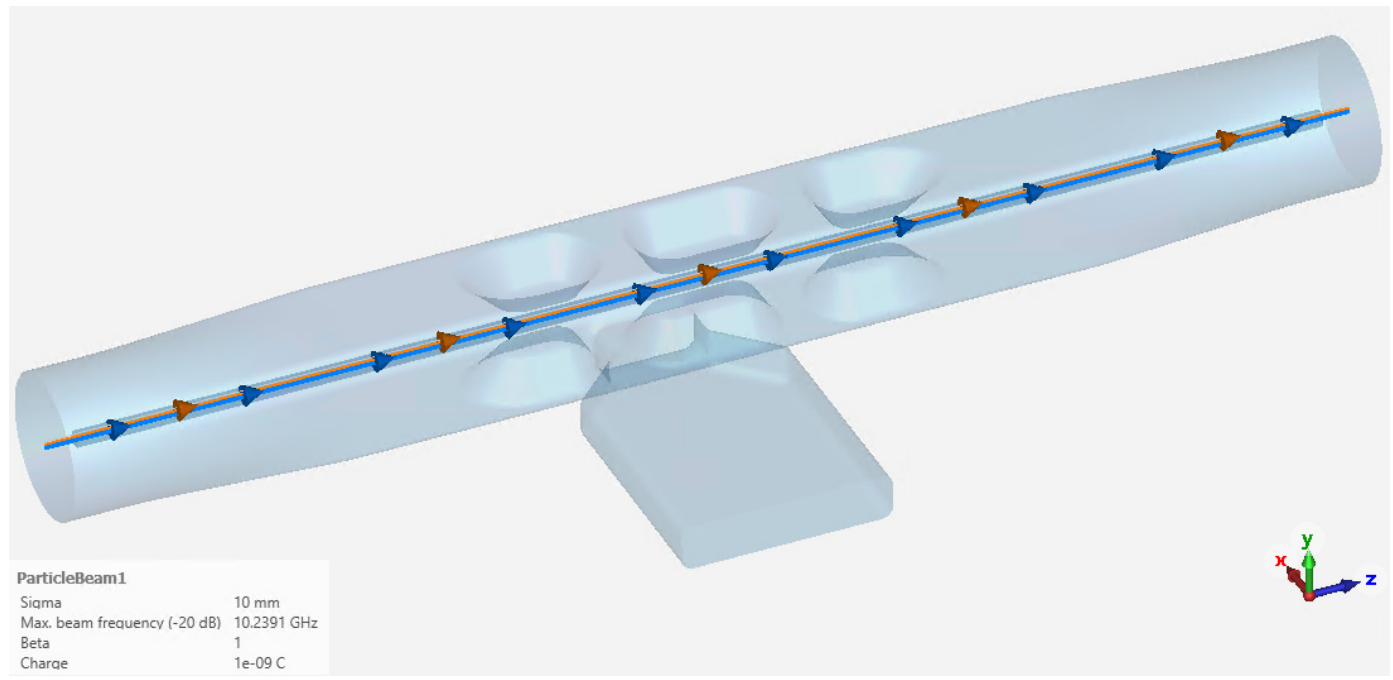

Figure 9. CST Particle Studio model of the deflecting cavity wakefield analysis.

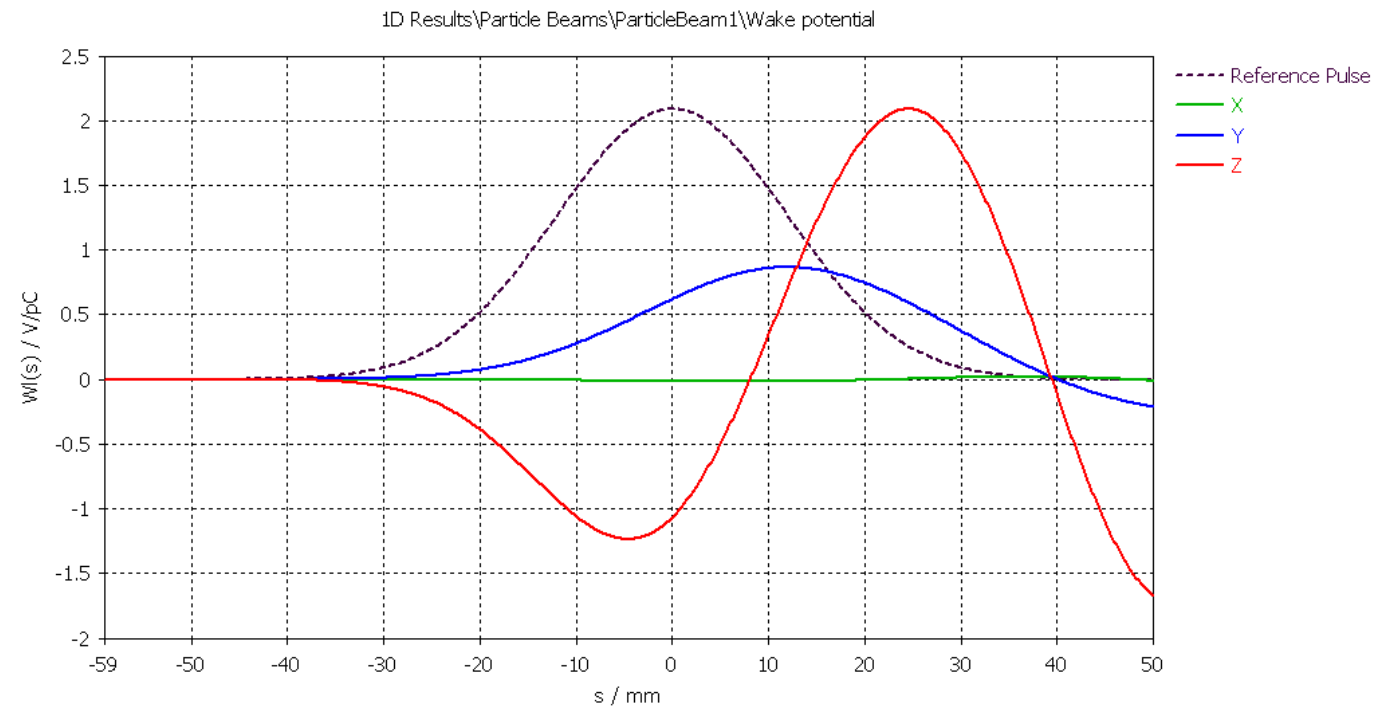

Figure 10. Calculated wake potentials exited by a relativistic Gaussian bunch with rms length of $10 \mathrm{~mm}$ passing through the 3.25-GHz cavity (variant A) with a vertical offset of $1 \mathrm{~mm}$.

In the time domain, the loss and kick factors are determined by convolution of the wake potential and the line density of the bunch profile as follows:

$$
\begin{aligned}
& \boldsymbol{k}_{\|}=\int_{-\infty}^{\infty}\left(W_{\|}(\boldsymbol{r}, \tau)\right)_{r=0} \lambda(\tau) d \tau, \\
& \boldsymbol{k}_{\perp}=\int_{-\infty}^{\infty}\left(W_{\perp}(\boldsymbol{r}, \tau)\right)_{r=r_{0}} \lambda(\tau) d \tau,
\end{aligned}
$$

where $\boldsymbol{W}_{\|}$and $\boldsymbol{W}_{\perp}$ are the longitudinal and transverse wake potentials, $r_{0}$ is the offset, and $\lambda(\tau)$ is the line density of the particle distribution [6]. Note that we calculate the transverse kick factors at a 1-mm offset. The results of wakefield simulations are presented in Figure 11 for variant A of the deflecting cavity. The decrease in the loss factor with frequency is explained by the fact that variant $\mathrm{A}$ is a scaled version of the 
QMiR cavity with the HOM spectrum shifted towards higher frequencies, while the spectrum of the 10-mm bunch interacting with the cavity remains constant and frequency limited.

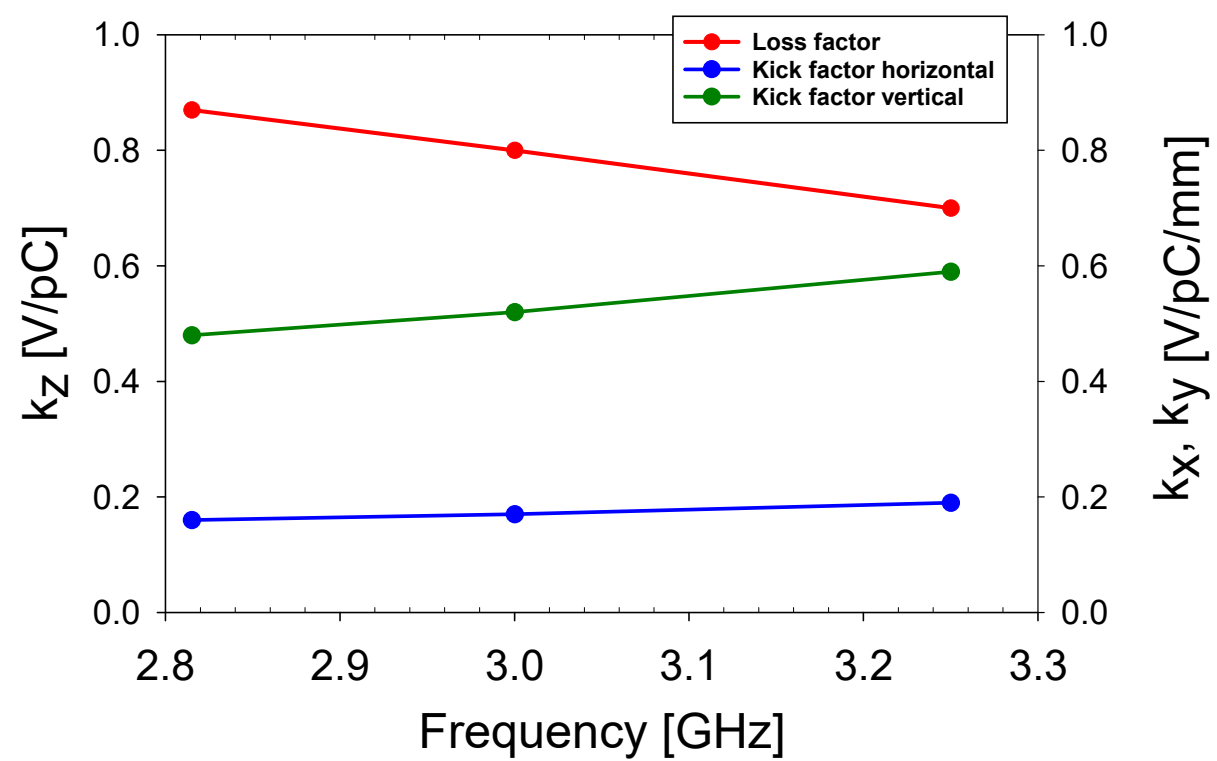

Figure 11. Loss factor (red) and kick factors (blue and green) of variant A of the deflecting cavities.

The vertical kick factor produced by a single variant A cavity is about $0.6 \mathrm{~V} / \mathrm{pC} / \mathrm{mm}$, which is enough for the total kick factor of a system consisting of even a minimum of three resonators to exceed the design limit of $1.3 \mathrm{~V} / \mathrm{pC} / \mathrm{mm}$. The remedy is to increase the cavity vertical beam aperture. Figure 12 illustrates the dependencies of the loss and kick factors on the vertical beam aperture in variant B of the deflecting cavity.

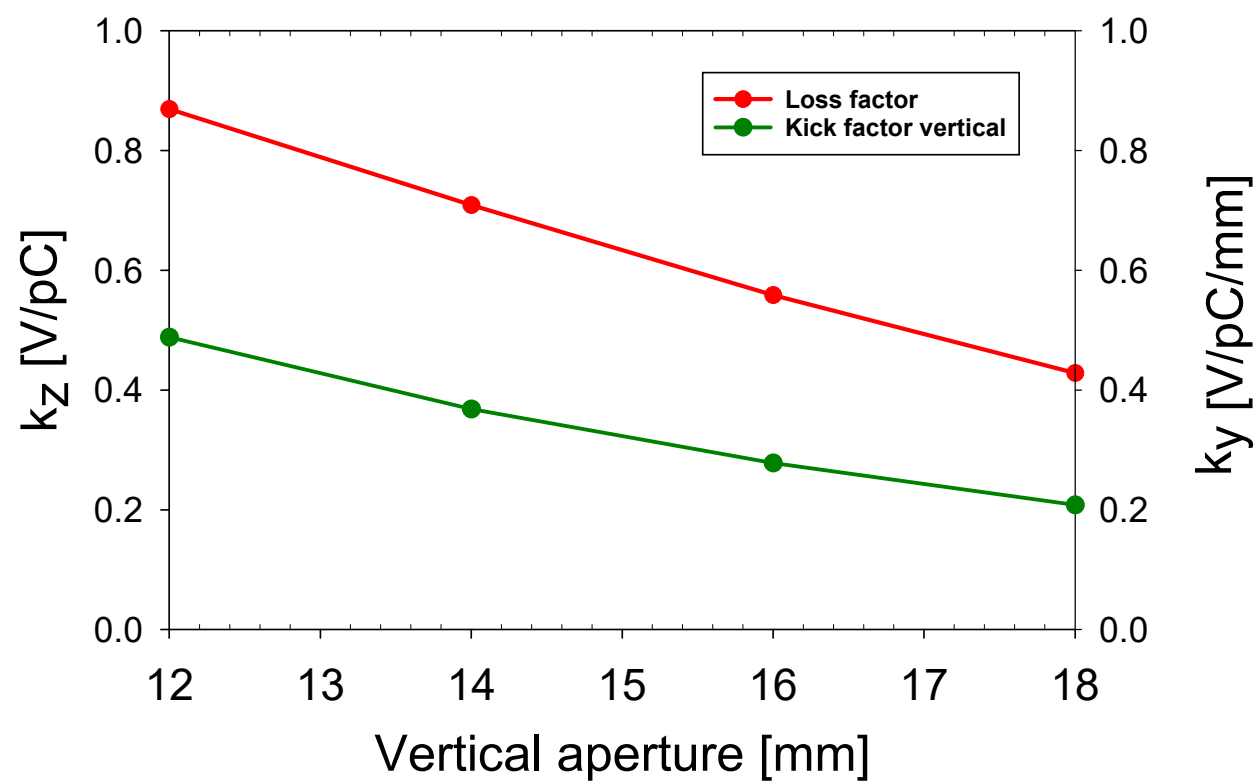

Figure 12. Loss factor (red) and vertical kick factor (green) of variant B of the deflecting cavities. 
Table 3 shows the summary of wakefield analyses for both variants A and B of the deflecting cavity for ST. Variant A of the deflection system meets the loss factor requirement but exceeds the vertical kick factor limit by $25 \%$. The alternative variant $\mathrm{B}$, with an increased vertical beam aperture, fully satisfies all design requirements for the excitation of short-range wakefields. It is also possible to further optimize variant $\mathrm{B}$ to improve the integral characteristics of the deflecting system.

Table 3. Loss and kick factors of variants A and B of the deflecting cavity for ST.

\begin{tabular}{|c|c|c|c|c|c|c|c|}
\hline \multirow{3}{*}{$\begin{array}{l}\text { Parameters of } \\
\text { Short Range } \\
\text { Wakefields }\end{array}$} & \multicolumn{3}{|c|}{ Variant A } & \multicolumn{3}{|c|}{ Variant B } & \multirow{3}{*}{$\begin{array}{l}\text { Design } \\
\text { Limit }\end{array}$} \\
\hline & \multicolumn{2}{|c|}{ Single cavity } & \multirow{2}{*}{ Total } & \multicolumn{2}{|c|}{ Single cavity } & \multirow{2}{*}{ Total } & \\
\hline & $3 \mathrm{GHz}$ & $3.25 \mathrm{GHz}$ & & $3 \mathrm{GHz}$ & $3.25 \mathrm{GHz}$ & & \\
\hline $\begin{array}{l}\text { Loss Factor, } \\
\mathrm{V} / \mathrm{pC}\end{array}$ & 0.8 & 0.7 & 2.2 & 0.7 & 0.5 & 2.4 & 4 \\
\hline $\begin{array}{l}\text { Horizontal } \\
\text { Kick Factor, } \\
\mathrm{V} / \mathrm{pC} / \mathrm{mm}\end{array}$ & 0.17 & 0.19 & 0.53 & 0.17 & 0.19 & 0.72 & 1.3 \\
\hline $\begin{array}{l}\text { Vertical } \\
\text { Kick Factor, } \\
\mathrm{V} / \mathrm{pC} / \mathrm{mm}\end{array}$ & 0.52 & 0.59 & 1.63 & 0.37 & 0.25 & 1.24 & 1.3 \\
\hline
\end{tabular}

\subsection{Parameters of the Operating Mode and Cavity Cryogenic Losses}

Operation of the SRF cavity requires a cryogenic system that can maintain the cavity under static and dynamic heat loads. While the static part is fixed and can be reliably predicted by the cryostat design, the dynamic load is dependent on the cavity voltage, operating temperature, and cavity material. Accordingly, the use of a deflection cavity at lower voltage and higher temperature is beneficial in terms of reducing both capital and operating costs of the cryogenic system. In addition, we assume the following cavity material options: niobium $(\mathrm{Nb})$, niobium doped with nitrogen, and a $\mathrm{Nb}_{3} \mathrm{Sn}$ compound. The pure niobium is the most technologically advanced material for SRF cavity production, but it suffers from mediocre surface resistance at temperatures above $2 \mathrm{~K}$. The recently developed niobium doped with nitrogen demonstrates a record low surface resistance at $2 \mathrm{~K}$. The $\mathrm{Nb}_{3} \mathrm{Sn}$ is a new experimental superconducting material with high critical temperature just over $18 \mathrm{~K}$, and consequently a lower surface resistance at $4 \mathrm{~K}$. Operating the cavity in the $4 \mathrm{~K}$ temperature range has the advantage of allowing a dry cooling scheme (no liquid helium) based on conduction cooling from commercial cryocoolers. For estimations of the materials' surface resistance, we use the experimental data measured by different research groups for the 3.9-GHz third-harmonic cavity of the LCLS-II project [7-9]. Scaling to operating frequencies of $3 \mathrm{GHz}$ and $3.25 \mathrm{GHz}$ is performed by the theoretical approximation of surface resistance for low temperatures [10]:

$$
R_{s}(\omega, T) \propto A\left(\omega^{2} / T\right) e^{-[\Delta(T) / k T]},
$$

where $A$ is the material-dependent constant, and $\Delta(\boldsymbol{T})$ is the superconducting energy gap. The estimated values of the material surface resistance at temperatures of $2 \mathrm{~K}$ and $4 \mathrm{~K}$ are presented in Table 4 for the operating parameters of the deflecting cavities. Note that we estimate the surface magnetic field to be below $20 \mathrm{mT}$, and the typical ambient magnetic field is about $10 \mathrm{mG}$. The latter has been demonstrated in cryomodules developed for LCLS-II using magnetic shielding around the accelerating cavity [11]. According to the tests of the 3-GHz elliptical cavity performed at JLab [12], one can expect to achieve a surface resistance of $\mathrm{Nb}$ at $2 \mathrm{~K}$ and $3 \mathrm{GHz}$ in the range $35-70 \mathrm{n} \Omega$. However, we also consider that the cavity string assembly is much longer than its transverse dimension, and it can be difficult to perform surface treatment procedures (electropolishing and high-pressure rinsing) to achieve the best performance, so we take a very conservative approach in our estimation of the surface resistance of materials. 
Table 4. Material surface resistances for operating conditions of deflecting cavities (surface magnetic field less than $20 \mathrm{mT}$ and ambient magnetic flux of $10 \mathrm{mG}$ ).

\begin{tabular}{|l|c|c|}
\hline \multirow{2}{*}{ Cavity Material } & \multicolumn{2}{|c|}{ Surface Resistance $(f 1 / f 2), \mathrm{n} \Omega$} \\
\cline { 2 - 3 } & $@ 2 \mathrm{~K}$ & @ $4.3 \mathrm{~K}$ \\
\hline $\mathrm{Nb}$ & $110 / 130$ & $820 / 960$ \\
\hline Nitrogen doped $\mathrm{Nb}$ & $50 / 60$ & $2500 / 2900$ \\
\hline $\mathrm{Nb}_{3} \mathrm{Sn}$ & - & $85 / 100$ \\
\hline
\end{tabular}

Another key parameter for calculating the cryogenic losses of the cavity is the geometric factor of the operating mode, defined as:

$$
G=\frac{\omega_{0} \mu_{0} \int_{V}|H|^{2} d v}{\int_{S}|H|^{2} d s}
$$

where $\mu_{0}$ is the vacuum permeability constant, and $H$ is the cavity magnetic field. The surface electric and magnetic fields of the operating mode are shown in Figure 13 for the scaled version of the 3-GHz deflecting cavity. Because the geometrical factor depends on the cavity shape only, it remains almost constant for all versions the deflecting cavity.
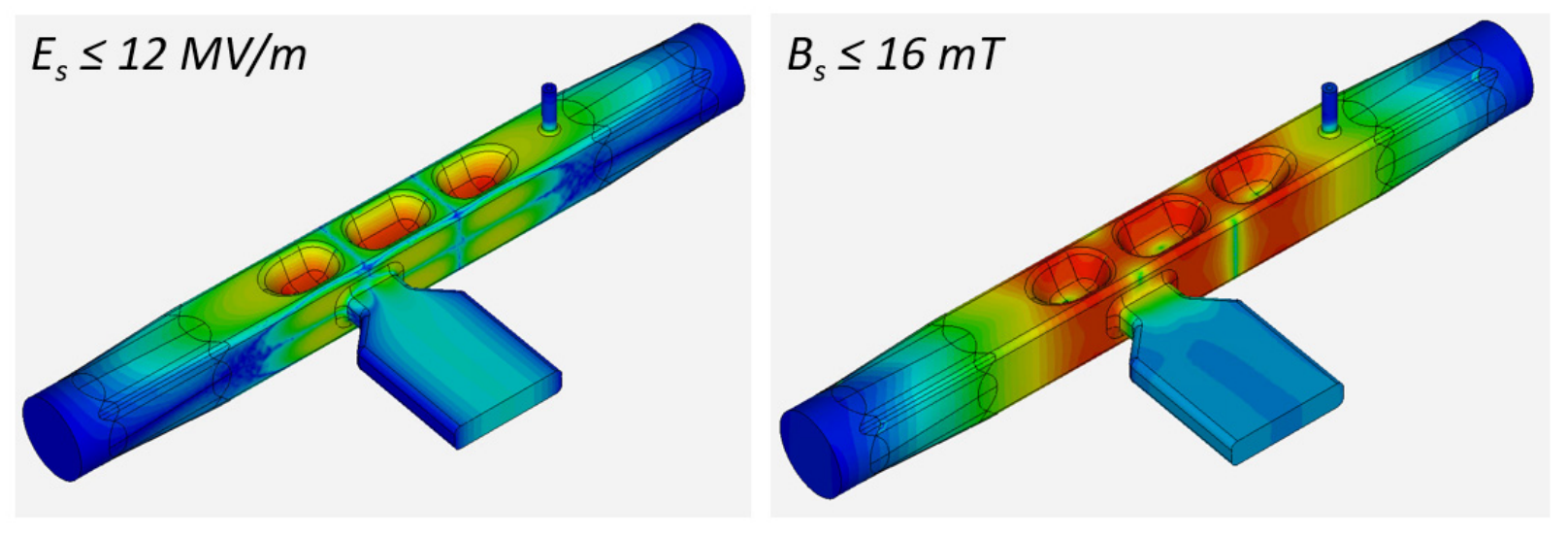

Figure 13. Surface electric (left) and magnetic (right) fields of the 3-GHz deflecting cavity (variant A) calculated for a vertical kick of 0.4 MV (logarithmic scale).

Finally, we can estimate the cavity cryogenic losses for a given vertical kick in each type of deflecting cavity as follows:

$$
P_{c}=\frac{\omega_{0} W_{0} R_{s}}{G}
$$

where $W_{0}$ is the operating mode nominal stored energy, and $R_{S}$ is the material surface resistance. The external coupling of the operating mode is a tunable parameter in the range of about $10^{5}$ to $10^{6}$ and is a trade-off between required RF input power and damping of the SOMs. The calculated parameters of the operating mode for various options of the proposed deflecting cavity are summarized in Table 5. The maximum surface electric and magnetic fields do not exceed $24 \mathrm{MV} / \mathrm{m}$ and $32 \mathrm{mT}$ for all configurations of 
the deflecting cavity. This is roughly equivalent to an $8-\mathrm{MV} / \mathrm{m}$ accelerating gradient in the $3.9-\mathrm{GHz}$ cavity for the LCLS-II, which is about half of the nominal operation gradient of the third-harmonic system. Here we can conclude that any of the proposed designs will operate at the required vertical kick voltage with a large margin of safety in terms of quench and field emission thresholds. The required RF input power will not exceed a few hundred watts and can probably be reduced below 100 watts by optimizing the variant $\mathrm{B}$ of the cavity. Note that the input power is estimated for the beam traveling along the cavity axis and does not consider the beam loading effect, which may require additional RF power depending on the cavity alignment and beam parameters.

Table 5. Parameters of the operating mode for different options of the deflecting cavity for ST.

\begin{tabular}{|c|c|c|c|c|c|c|c|c|c|c|}
\hline \multirow{2}{*}{ Variant } & \multirow{2}{*}{$\begin{array}{l}\text { Freq. } \\
\mathrm{GHz}\end{array}$} & \multirow{2}{*}{$\begin{array}{c}\text { Vertical } \\
\text { Kick } \\
\text { MV }\end{array}$} & \multirow{2}{*}{$\begin{array}{l}(R / Q)_{y}, \\
\mathrm{k} \Omega / \mathrm{m}\end{array}$} & \multirow{2}{*}{$\begin{array}{c}\mathrm{E}_{\max } \\
\mathrm{MV} / \mathrm{m}\end{array}$} & \multirow{2}{*}{$\begin{array}{l}\mathrm{B}_{\max } \\
\mathrm{mT}\end{array}$} & \multirow{2}{*}{$G$} & \multicolumn{3}{|c|}{ RF Loss $(2 \mathrm{~K} / 4 \mathrm{~K}), \mathrm{W}$} & \multirow{2}{*}{$\begin{array}{c}\text { Pinp } \\
\text { W }\end{array}$} \\
\hline & & & & & & & $\mathrm{Nb}$ & $\begin{array}{l}\text { Nitrogen- } \\
\text { doped } \mathrm{Nb}\end{array}$ & $\mathrm{Nb}_{3} \mathrm{Sn}$ & \\
\hline \multirow{2}{*}{ A } & 3.0 & 0.8 & 32.9 & 24 & 32 & \multirow{4}{*}{142} & $0.5 / 3.6$ & $0.2 / 11$ & $-/ 0.4$ & 60 \\
\hline & 3.25 & 0.37 & 35.6 & 12 & 16 & & $0.1 / 0.9$ & $0.05 / 2.8$ & $-/ 0.1$ & 50 \\
\hline \multirow{2}{*}{ B } & 3.0 & 0.4 & 20.1 & 14 & 20 & & $0.2 / 1.5$ & $0.1 / 4.5$ & $-/ 0.15$ & 300 \\
\hline & 3.25 & 0.37 & 10.7 & 18 & 27 & & $0.4 / 3.0$ & $0.2 / 9.0$ & $-/ 0.2$ & 320 \\
\hline
\end{tabular}

The total expected cryogenic losses are shown in Table 6 for both variants A and B of the deflecting system. The overall cavity dynamic loss for operation at $2 \mathrm{~K}$ temperature is about $1 \mathrm{~W}$, which is comparable to expected static conducting heat loads from the couplers and beam pipe. Thus, pure $\mathrm{Nb}$ is the obvious choice for cavity fabrication, and there is no advantage to using the complex nitrogen-doping surface treatment. For $4 \mathrm{~K}$ operation, $\mathrm{Nb}$ appears to be acceptable to run the deflecting system with multiple cryocoolers. The $\mathrm{Nb}_{3} \mathrm{Sn}$ material can provide minimal losses at $4 \mathrm{~K}$ of less than $1 \mathrm{~W}$, which therefore allows operation with a single cryocooler system, but will require experimental confirmation of reliable work at the required nominal voltages.

Table 6. Total cryogenic losses of the proposed variants A (10.4-mm aperture) and B (13.9-mm aperture) of the deflecting system for ST.

\begin{tabular}{|c|c|c|c|}
\hline \multirow{2}{*}{$\begin{array}{c}\text { Temperature } \\
\text { Operating }\end{array}$} & \multicolumn{3}{|c|}{ Total Cryogenic Loss (A/B), W } \\
\cline { 2 - 4 } & $\mathrm{Nb}$ & Nitrogen-doped Nb & $\mathrm{Nb}_{3} \mathrm{Sn}$ \\
\hline $2 \mathrm{~K}$ & $0.7 / \mathbf{1 . 2}$ & $0.3 / 0.6$ & N/A \\
\hline $4 \mathrm{~K}$ & $5.2 / \mathbf{9 . 0}$ & N/A & $0.6 / \mathbf{0 . 7}$ \\
\hline
\end{tabular}

The uniformity of the operating deflection mode can be estimated by expanding the transverse momentum kick in terms of the azimuthal multipoles as follows [13]:

$$
\Delta p_{\perp}(r, \varphi)=q \sum_{n=1}^{N} r^{n-1}\left[b_{n} \cos (n \varphi)+a_{n} \sin (n \varphi)\right]
$$

where $N$ is the order of azimuthal sampling at radius $r$, and $a_{n}$ and $b_{n}$ are the skew and normal multipolar expansion coefficients, respectively. Using the Panofsky-Wenzel theorem, one can represent the expansion coefficients by a Fourier transformation of the longitudinal component of the electric field: 


$$
\begin{aligned}
& a_{n}=\frac{i n}{\omega \pi r^{n}} \int_{0}^{2 \pi} \sin (n \varphi) \int_{-\infty}^{\infty}\left(E_{z}(r, z, \varphi)\right)_{r=r_{0}} e^{i k z} d z d \varphi \\
& b_{n}=\frac{i n}{\omega \pi r^{n}} \int_{0}^{2 \pi} \cos (n \varphi) \int_{-\infty}^{\infty}\left(E_{z}(r, z, \varphi)\right)_{r=r_{0}} e^{i k z} d z d \varphi
\end{aligned}
$$

Based on equations (8) and (9), we calculated the multipole expansion coefficients for the 3.25-GHz cavity (variant B) at a radial distance of $4 \mathrm{~mm}$. The results are presented in Table 7 . Here the deflecting cavity has the nominal transverse voltage of $0.37 \mathrm{MV}$ determined by the first-order normal coefficient $b_{1}$. The skew sextuple RF field component is determined by the $a_{3}$ coefficient of maximum $0.16 \mathrm{Tm} / \mathrm{m}^{2}$ and is below the design requirements of $4 \mathrm{Tm} / \mathrm{m}^{2}\left(120 \mathrm{kV} / \mathrm{cm}^{2}\right)$. Further reduction of both normal and skew sextuple components is possible by flattening the inner surface of the electrodes $[14,15]$.

Table 7. Normal and skew multipolar components of the 3.25-GHz (variant B) deflecting cavity calculated at radial distance of $4 \mathrm{~mm}$ and nominal transverse voltage of $0.37 \mathrm{MV}$.

\begin{tabular}{|c|c|c|c|c|c|}
\hline \multicolumn{7}{|c|}{ Multipole Coefficients } \\
\hline \multicolumn{3}{|c|}{ Normal } & \multicolumn{3}{c|}{ Skew } \\
\hline $\begin{array}{c}\left|\boldsymbol{b}_{1}\right|, \\
\mathrm{Tm}\end{array}$ & $\left|\boldsymbol{b}_{2}\right|$, & $\begin{array}{c}\boldsymbol{b}_{3} \mid, \\
\mathrm{Tm} / \mathrm{m}\end{array}$ & $\begin{array}{c}\left|\boldsymbol{a}_{\mathbf{1}}\right|, \\
\mathrm{Tm} / \mathrm{m}^{2}\end{array}$ & $\begin{array}{c}\boldsymbol{a}_{2} \mid, \\
\mathrm{Tm} / \mathrm{m}\end{array}$ & $\begin{array}{c}\mathrm{T} \\
\mathrm{Tm} / \mathrm{m}^{2}\end{array}$ \\
\hline $1.3 \times 10^{-3}$ & $2.8 \times 10^{-4}$ & 9.4 & $5.8 \times 10^{-7}$ & $8.9 \times 10^{-4}$ & 0.16 \\
\hline
\end{tabular}

\subsection{Estimations of the Cost and Duration of the Cavity EM and Mechanical Design Work}

The suggested RF deflection system based on the SRF cavities requires the cavities, cooling system, cryomodule, RF power system, HOM/SOM dampers, and various vacuum and mechanical components. In the first step, a cavity concept should be developed, and the key technical requirements must be met: cavities layout, external coupling bandwidth, RF power requirements, and HOM issues. The overall length of the cavity string is about $1.2 \mathrm{~m}$, so all cavities can be fabricated as one piece from a single $\mathrm{Nb}$ rod. Figure 14 shows the welded assembly of a single QMiR niobium prototype that is $0.5 \mathrm{~m}$ long. In this case, a machining tolerance of less than $10 \mu \mathrm{m}$ is easily achieved, which greatly simplifies the cavity alignment system.

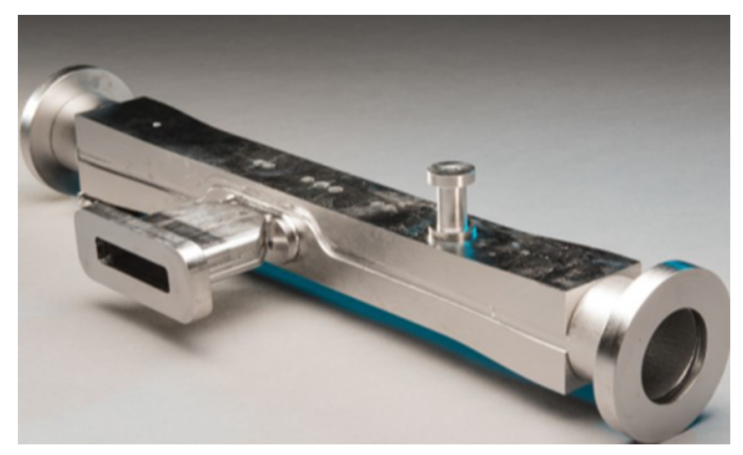

Figure 14. A QMiR cavity niobium prototype.

Because of the nature of the cavity design, the HOM signal is freely radiated out of the cavity and can be absorbed by the walls of a vacuum chamber, usually made of lossy stainless steel. In the case of existing sensitive equipment before or after the deflection system, a Beam Line Absorber (BLA) can be installed 
upstream and downstream to damp the radiated RF power of the HOMs. Typically, a BLA is made of lossy ceramic and is capable of absorbing hundreds of watts when properly cooled [16]. SOM damping is provided by the waveguide input port, which in turn will require the development of a broadband RF window and a dedicated RF absorbing circuit. In order to compensate for the frequency uncertainty after cooling the cavity and to maintain resonance during operation, it is necessary to have at least a coarse frequency tuner. A fine frequency tuner is likely not required given the relatively large, anticipated cavity bandwidth due to external coupling of less than $10^{6}$. A general scheme of the proposed deflecting system is illustrated in Figure 15. The overall length of the system will not exceed $2 \mathrm{~m}$ and can be reduced to $1.5 \mathrm{~m}$ without installation of a BLA.

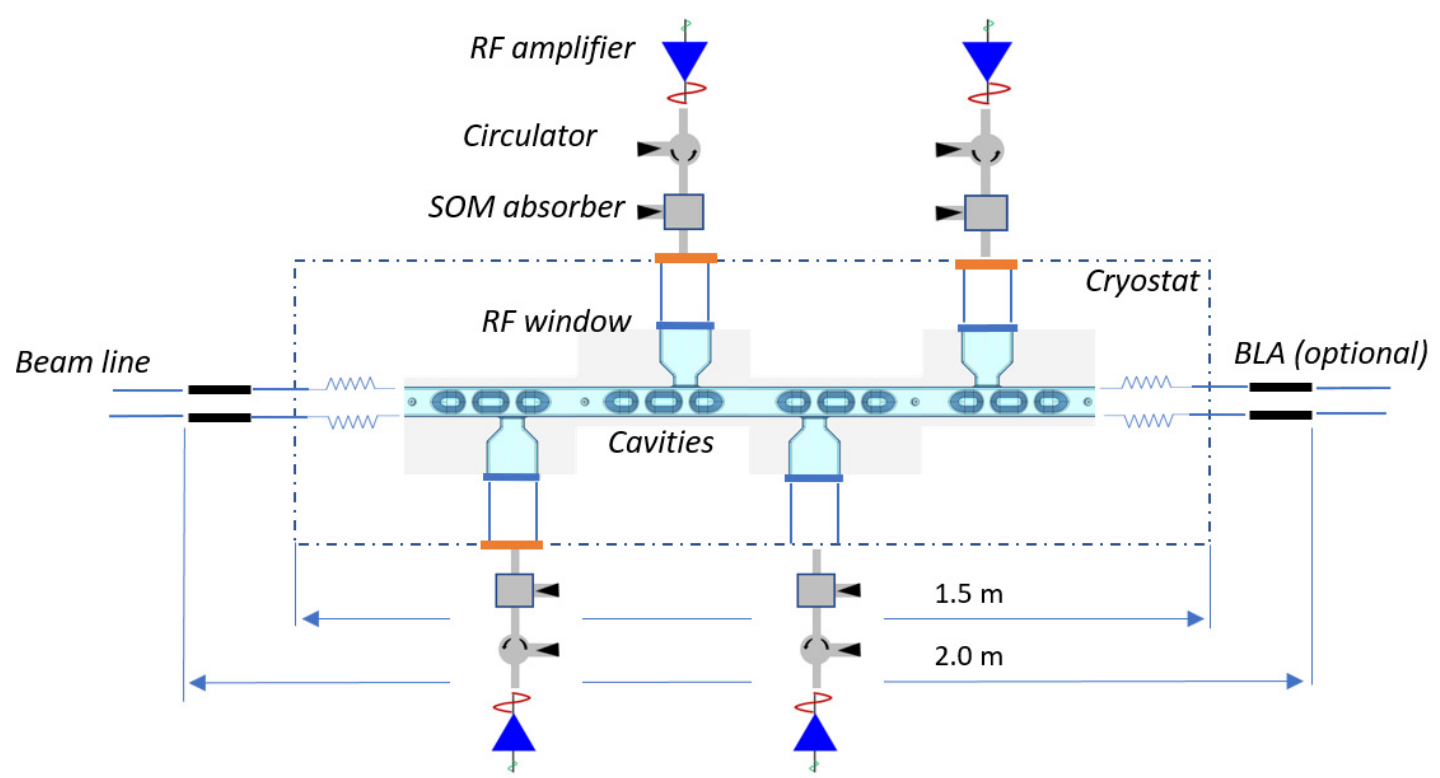

Figure 15. General scheme of the proposed deflecting system for the ST SPX project.

To design the entire deflection system, one must understand whether a cryogenic system is available in the workplace. If yes, a He vessel should be designed. Otherwise, cryocoolers may be used to operate with a cavity at $4 \mathrm{~K}$. A conventional $\mathrm{Nb}$ cavity processing $(\mathrm{BCP})$ requires four to five cryocoolers, each with a capacity of $2.5 \mathrm{~W}$. The typical cost of such a cryocooler is about $\$ 80 \mathrm{k}$. In this case, instead of a LHe vessel, a conductive cooling system should be designed. Similar cooling schemes have recently been developed and successfully tested for single-cell cavities at Fermilab, Cornell, and JLab [17-19]. Figure 16 shows conductive cooling straps mounted on a 2.6-GHz cavity at Cornell. The adaptation of such a system to the ST deflecting cavities looks straightforward, thanks to the flat surfaces of the outer walls of the cavity.

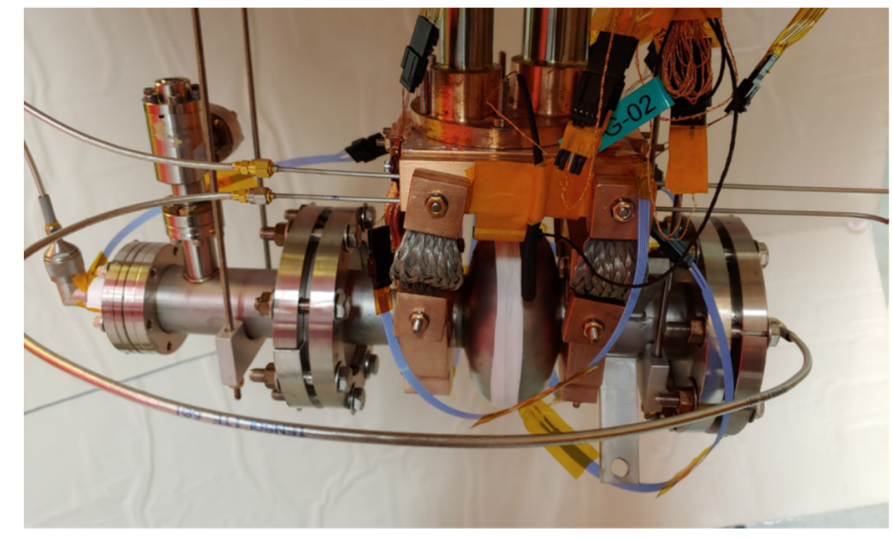

Figure 16. Conduction cooling system installed on the 2.6-GHz cavity at Cornell. 
Most of the cost of an RF system is determined by the cost of an amplifier with the required output power of several hundred watts. Conservatively, we expect the $0.5-\mathrm{kW} \mathrm{CW} 3-\mathrm{GHz}$ and $3.25-\mathrm{GHz}$ amplifiers to cover all deflecting cavity operation scenarios with sufficient margin. Typical prices for commercially available broadband amplifiers of this type are around $\$ 100,000$ each. The cost of a custom narrowband amplifier can be significantly cheaper.

This project requires two types of cavities. At first glance, the designs are similar, but have slightly different shapes and dimensions. Therefore, for each cavity, a separate optimization of the electromagnetic (EM) field is required (minimization of surface field losses, wake and HOM mitigation, tolerance analysis, etc.). The cavity design needs multipacting (MP) analysis, which may affect the shape of the cavity. In fact, EM and MP optimization are self-consistent and iterative processes. In order to avoid the cavity quench, a detailed thermal analysis is required. Mechanical analysis of the cavity, including a $\mathrm{He}$ vessel or a conductive cooling system, is necessary to mitigate microphonics and Lorentz Force Detuning (LFD), and to meet safety codes. A cavity coarse frequency tuner should be developed, and fine tuners should be designed if needed. Designs of the RF circuit for damping SOMs and a possible BLA for HOMs are required. The estimated cost of a self-consistent EM-MP thermal and mechanical design for both cavities is 2 FTEs. The total duration of the design part of the project is about one year. A compact cryostat should be designed including a cavity support system, thermal and magnetic shields, cryogenics, piping, an alignment system, RF input, connectors, valves, etc., which will take 0.5 FTE. To extract SOMs, an external RF circuit needs to be designed, which will take 0.1 FTE. In addition, a HOM damping system may require BLA development, which will take 0.1 FTE. Considering vacuum/mechanical parts, LLRF, and installation, the total is 4.2 FTEs. A preliminary cost estimate is summarized in Table 8. Note that this estimate was made for the $\mathrm{Nb}$ resonator, the technological process of which has been matured and does not require prototyping. R\&D work and prototyping of the $\mathrm{Nb}_{3} \mathrm{Sn}$ cavity will require additional funding and should be evaluated separately.

Table 8. Superconducting deflecting cavity system for ST cost estimates

\begin{tabular}{|l|c|c|}
\hline Components & $\begin{array}{c}\text { Labor, } \\
\text { FTE }\end{array}$ & $\begin{array}{c}\text { M\&S, } \\
\text { M\$ }\end{array}$ \\
\hline Deflecting cavities & 2 & 0.5 \\
\hline Compact cryostat & 0.5 & 0.5 \\
\hline $\begin{array}{l}\text { Cryogenic system } \\
\text { (4K, 5 dilution cryocoolers @2.5 W each) }\end{array}$ & 0.1 & 0.4 \\
\hline $\begin{array}{l}\text { RF power system } \\
\text { 4 SSA @500 W CW each) }\end{array}$ & 0.1 & 0.5 \\
\hline $\begin{array}{l}\text { Input couplers } \\
\text { (4 ceramic windows with cooling) }\end{array}$ & 0.2 & 0.1 \\
\hline SOM/HOM dampers & 0.2 & 0.2 \\
\hline Vacuum/Mechanical parts & 0.1 & 0.2 \\
\hline LLRF and controls & 0.5 & 0.3 \\
\hline Installation & 0.5 & 0.2 \\
\hline & & $\mathbf{4 . 2}$ \\
\hline Total & $\mathbf{2 . 8}$ \\
\hline
\end{tabular}


The total amount of work requires is 4.2 FTEs and an M\&S cost of $\$ 2.8$ million. The time necessary to design, build, and test the entire deflecting system with an RF station is about two years. These cost estimates do not include any contingency and have not been subjected to a complete bottom-up assessment or review. More accurate estimates may result in significant changes.

\subsection{Anticipated Cost of Operation and Maintenance of the Deflecting Cavity System}

The cavities for this project do not need maintenance. On the other hand, the cryogenic system will require a significant maintenance cost. Five $2.5-\mathrm{W}$ cryocoolers will be adequate for cooling cavities manufactured using the existing standard $\mathrm{Nb}$ technology. One cryocooler may be enough for cooling cavities coated with $\mathrm{Nb}_{3} \mathrm{Sn}$. The typical efficiency of a state-of-the-art $4 \mathrm{~K}$ cryocooler is about $2 \times 10^{-4}$; thus, each cryocooler consumes a maximum $12.5 \mathrm{~kW}$ of electrical power from the wall plug [20]. The yearly maintenance cost for each cryocooler is about $\$ 10 \mathrm{k}$. For a $2 \mathrm{~K}$ refrigerator, the maintenance cost depends strongly on the cooling capacity and the exact situation with a cryo-distribution system; this must be estimated elsewhere.

Note that for the SRF cavity the cost of electricity is small, because the power needed to maintain an $\mathrm{RF}$ field is quite small, well below $1 \mathrm{~kW}$. The maintenance cost for each RF amplifier usually does not exceed \$10k per year [21]. Therefore, the overall operational cost necessary to run the cavity with cryocoolers is about $\$ 110 \mathrm{k}$ per year, considering an electricity cost of $\$ 0.2 / \mathrm{kW}-\mathrm{h}$. For a refrigerator, the efficiency is substantially higher-about $10^{-3}$; this cost is about $\$ 20 \mathrm{k}$ per year. The total expected operational and maintenance expenses are presented in Table 9. The data for the $2 \mathrm{~K}$ refrigerator is for comparison only and is not included in the estimate of total wall plug power and total costs. Here we assume 12-month operation per year for 8760 hours total.

Table 9. Operational and maintenance cost of the superconducting deflecting cavity system.

\begin{tabular}{|c|c|c|c|c|}
\hline Key components & $\begin{array}{c}\text { Efficiency, } \\
\%\end{array}$ & $\begin{array}{c}\text { Wall plug } \\
\text { power, kW }\end{array}$ & $\begin{array}{l}\text { Operational } \\
\text { cost }^{1}, \mathrm{k} \$ / \text { year }\end{array}$ & $\begin{array}{l}\text { Maintenance } \\
\text { cost, } \mathrm{k} \$ \text { /year }\end{array}$ \\
\hline $\begin{array}{l}\text { Cryogenic system } \\
(2 \mathrm{~K}, \mathrm{LHe} \text { refrigerator, } \\
(\text { a } 10 \mathrm{~W})^{2}\end{array}$ & 0.1 & 10 & 17.5 & N/A \\
\hline $\begin{array}{l}\text { Cryogenic system } \\
\text { ( } 4 \mathrm{~K}, 5 \text { dilution cryocoolers } \\
@, 2.5 \mathrm{~W} \text { each) }\end{array}$ & 0.02 & 62.5 & 110 & 50 \\
\hline $\begin{array}{l}\text { RF power system } \\
\text { (4 SSA@500 W CW each) }\end{array}$ & 30 & 7 & 12 & 40 \\
\hline Total & & $\sim 70 \mathrm{~kW}$ & \multicolumn{2}{|c|}{$\sim 210 \mathrm{k \$} /$ year } \\
\hline
\end{tabular}

${ }^{1}$ assumes 12 -month operation per year $(8760 \mathrm{~h})$ at $\$ 0.2 / \mathrm{kW}-\mathrm{h}$

${ }^{2}$ data for a $2 \mathrm{~K}$ refrigerator is for comparison only

\subsection{Final Remarks and Conclusion}

For the Short Pulse X-ray project at Synchrotron Trieste, Fermilab proposes a compact SRF cavity, QMiR, which has been invented and designed for the ANL Advanced Photon Source (APS). The cavity contains four short $(<40 \mathrm{~cm}$ long) sections. Two of them operate at a frequency of $3 \mathrm{GHz}$, and the other two at $3.25 \mathrm{GHz}$. The total length of the deflecting structure may be about $1 \mathrm{~m}$, or even less, which makes it possible to machine all cavities a whole and greatly simplifies alignment. The cavity aperture can be made 
the same as the ring aperture. The total length of a cryostat including flanges, bellows, and valves, may be less than 1.4-1.5 m. A preliminary concept design of the cavity demonstrates that the required parametersdeflecting voltage, HOM impedance, and wakefields - can be achieved even after a brief optimization. The cavity is very compact, simple, and inexpensive. The RF power requirement is extremely modest, well below $1 \mathrm{~kW}$. It is feasible that all four cavities may be cooled conductively with the use of four or five $2.5-\mathrm{W}$ cryocoolers, which are commercially available at about $\$ 80 \mathrm{k}$ each. With some modest investment in $\mathrm{Nb} 3 \mathrm{Sn}$ technology, the result can potentially lead to a significant reduction of $4 \mathrm{~K}$ heat load, and a single cryocooler may be sufficient to operate the system.

Note that SRF is a well established and mature technology. Fermilab has extensive experience in the development of SRF resonators for accelerator applications - accelerating and deflecting cavities. Fermilab has an experienced team and state-of-the-art facilities to support the entire development cycle of SRF systems: beam dynamics analysis, RF design, manufacturing, processing, testing and commissioning. Over the past 20 years, Fermilab has developed SRF cavities with a wide frequency range from $325 \mathrm{MHz}$ to 3.9 GHz. Fermilab recently designed and built twenty $1.3 \mathrm{GHz}$ and three $3.9 \mathrm{GHz}$ cryomodules for the LCLS-II light source at SLAC and over ten SRF crab cavities for the HL-LHC project at CERN. We are currently building and commissioning several cryomodules of different types for the Fermilab $800 \mathrm{MeV}$ linear proton accelerator, PIP II. We are fully confident that the modified QMIR cavity will operate successfully in the Trieste SPX project.

\subsection{Acknowledgements}

The authors are thankful to Dr. G. Eremeev for providing critical information on surface resistivity for various types of niobium treatment and for discussing possible cavity processing options; Mr. Dhuley for information on cooling options; Mr. R. Pilipenko for cost estimates and maintenance of the RF system; and Prof. S. Belomestnykh for carefully reading the manuscript and providing many critical remarks.

\section{References}

1. A. Zholents, P. Heimann, M. Zolotorev, J. Byrd, "Generation of subpicosecond X-ray pulses using RF orbit deflection," Nuclear Instruments and Methods in Physics Research Section A 425(1-2), 385-389 (1999).

2. A. Lunin, I. Gonin, M. Awida, T. Khabiboulline, V. Yakovlev, A. Zholents, "Design of a Quasiwaveguide Multicell Deflecting Cavity for the Advanced Photon Source," Physics Procedia 79, 54-62 (2015).

3. M. Awida, I. Gonin, A. Lunin et al., "Analysis of a Quasi-waveguide Multicell Resonator for SPX," WEPTY018, IPAC'15, Richmond, VA, USA, 2015.

4. Z.A. Conway et al., "Development and Test Results of a Quasi-waveguide Multicell Resonator," WEPRI050, IPAC'14, Dresden, Germany, 2014.

5. ANSYS, Inc., http://www.ansys.com/Products/Electronics/ANSYS-HFSS (2020).

6. B.W. Zotter and S.A. Kheifetz, Impedances and Wakes in High-Energy Particle Accelerators, World Scientific Publishing, 1997.

7. G. Ciovati, Ph.D. Thesis, Old Dominion University, Norfolk, 2005.

8. R. D. Porter, M. Liepe, J. T. Maniscalco, "High Frequency Nb ${ }_{3}$ Sn Cavities," MOP011, SRF2019, Dresden, Germany, 2019.

9. M. Martinello, M. Checchin, A. Romanenko et al., "Field-Enhanced Superconductivity in HighFrequency Niobium Accelerating Cavities," Phys. Rev. Lett. 121, 224801 (2018).

10. D. C. Mattis and J. Bardeen, Phys. Rev. 111, 412 (1958). 
11. S. Posen et al., "Role of magnetic flux expulsion to reach $\mathrm{Q}_{0}>3 \times 10^{10}$ in SRF cryomodules," Phys. Rev. Accel. Beams 22, 032001 (2019).

12. G. Ciovati, G. Eremeev, and F. Hannon, "High field Q slope and the effect of low-temperature baking at 3 GHz," Phys. Rev. Accel. Beams 21, 012002 (2018).

13. J. Barranco Garcia et al., "Long term dynamics of the high luminosity Large Hadron Collider with crab cavities," Phys. Rev. Accel. Beams 19, 101003 (2016).

14. S. U. De Silva, Ph.D. Thesis, Old Dominion University, Norfolk, 2014.

15. A. A. Castilla, J. R. Delayen, "Multipole and Field Uniformity Tailoring of a $750 \mathrm{MHz}$ rf Dipole," MOPP117, LINAC2014, Geneva, Switzerland, 2014.

16. R. Eichhorn et al., "Higher Order Mode Absorbers for High Current ERL Applications," THBA05, SRF2015, Whistler, BC, Canada, 2015.

17. R. Dhuley et al., "First demonstration of a cryocooler conduction cooled superconducting radiofrequency cavity operating at practical cw accelerating gradients," Supercond. Sci. Technol. 33(6), 06LT01 (2020).

18. N. Stilin et al., "Stable CW Operation of $\mathrm{Nb}_{3} \mathrm{Sn}$ SRF Cavity at $10 \mathrm{MV} / \mathrm{m}$ using Conduction Cooling," arXiv:2002.11755v1 [physics.acc-ph] 26 Feb 2020.

19. G. Ciovati et al., "Multi-metallic conduction cooled superconducting radio-frequency cavity with high thermal stability," Supercond. Sci. Technol. 33(7), 07LT01 (2020).

20. R. Dhuley, Fermilab, private communication.

21. R. Pilipenko, Fermilab, private communication. 